\title{
Mise en scène de l'histoire sur les réseaux sociaux, pratiques et limites
}

\author{
Martin Grandjean
}

\section{Résumé}

Cet article propose un état des lieux des pratiques de médiation historique sur les réseaux sociaux numériques. Alors que les formes de communication autour d'événements, de fonds d'archives ou de thématiques historiques se multiplient sur ces plateformes, nous développons une typologie distinguant les différents usages des sources, dans le cadre de projets menés par des institutions patrimoniales ou de recherche comme dans des situations qui voient l'irruption d'acteurs aux apports discutables.

\section{Summary}

\section{History Depiction on Social Media: Practices and Limits}

This paper presents an inventory of historical mediation practices on social media. While the communications on historical events, archives or themes are multiplying on these platforms, we develop a typology distinguishing different uses of the sources, within the framework of projects led by heritage or research institutions as in situations where we observe the irruption of less scrupulous actors. 


\section{Quelle place pour l'histoire sur les}

\section{réseaux sociaux numériques?}

Depuis leur création, les plateformes de médias sociaux $^{1}$ sont le théâtre de nombreuses expérimentations en matière de médiation historique, qu'il s'agisse de projets conduits par des acteurs académiques, des institutions patrimoniales ou des médias. Or, ces protagonistes traditionnels de l'«histoire publique » sont aujourd'hui très largement dépassés par des initiatives contestables, des comptes créés pour partager automatiquement et massivement des photographies historiques, portraits célèbres et autres citations flatteuses à des millions d'abonnés sans s'encombrer de contextualisation, de licence, de véracité et de référencement des sources. C'est l'un des revers de la désintermédiation pourtant apparemment si fascinante apportée par les réseaux sociaux numériques au tournant du $\mathrm{XXI}^{\mathrm{e}}$ siècle : s'il est désormais possible à un passionné d'histoire de s'affranchir des acteurs officiels pour partager à une communauté d'abonnés des images ou événements qui lui tiennent à cœur ou dont il a acquis une certaine maitrise, nombreux sont ceux qui ont compris à quel point cette nouvelle forme de storytelling historique a trouvé un public particulièrement curieux et avide de retweeter l'une ou l'autre image d'archive rendant compte de la grande histoire de l'humanité ou de participer à la

\footnotetext{
${ }^{1}$ La plupart des exemples que nous proposons dans cet article sont issus de Twitter parce que la grande ouverture de cette plateforme permet une quantification plus aisée du phénomène et une plus grande appropriation de ce média par les chercheurs. Les hypothèses n'en sont pas moins valables pour Facebook et, dans une certaine mesure, Instagram. Nous n'abordons pas la diffusion de contenu historique sur YouTube, dont la couche «sociale» est trop récente.
}

commémoration d'un événement ${ }^{2}$ qui fonde une identité ou résonne avec une certaine actualité. Se pose dès lors la question de savoir si, dans un contexte où la plupart des contenus historiques qui inondent les principales plateformes sociales le sont à cause d'une course à l'audience parfois monétisée, une approche critique des sources reste possible ${ }^{3}$.

Cet article, qui renverse la perspective d'un «temps long des réseaux sociaux numériques» pour questionner la présence du temps long luimême dans ces médias pourtant réputés pour leur immédiateté, se présente sous la forme d'un état des lieux des pratiques et propose une typologie de l'usage des sources historiques sur le web social. Si cette synthèse peut être d'un certain intérêt pour des institutions académiques ou patrimoniales qui réfléchissent à leur présence en ligne, nous ne souhaitons toutefois pas en limiter la focale à ces utilisations. En effet, la montée en puissance d'acteurs commerciaux dont la stratégie de publicité, d'achat d'abonnés et d'autoréférencement multi-plateforme change profondément le paysage de la médiation culturelle sur les réseaux sociaux numériques a des implications sur les pratiques des protagonistes traditionnels. Ou du moins devrait-elle en avoir puisque cette concurrence pourrait bien avoir pour

\footnotetext{
${ }^{2}$ Voir la contribution de Frédéric Clavert dans ce numéro ainsi que F. Clavert, « Echos du centenaire de la Première Guerre mondiale sur Twitter », Matériaux pour l'histoire de notre temps, 121-122, 2016, p. 18-25

${ }^{3}$ Nous avons déjà abordé la question de la médiation de l'histoire sur Twitter, voir M. Grandjean, «Source criticism in 140 characters: rewriting history on social networks », International Federation for Public History Conference, Amsterdam, 2014 et M. Grandjean, « Médias sociaux et mise en scène de l'histoire », Digital Humanities, Montréal, 2017, p. 458-459. Voir également S. Noiret, «Trabajar con el pasado en internet: la historia pública digital y las narrativas de las redes sociales », Ayer, 110 (2), 2018, p. 111-140.
} 
conséquence une prise de conscience de l'intérêt que porte le public pour des formes de médiation historique qui tiennent parfois plus du récit et de la mise en scène que du simple partage de ressources. De fait, intégrer dans cette analyse les pratiques prédatrices, parfois commerciales et souvent motivées par une quête d'influence, c'est également tenter de comprendre de quelle manière mettre à profit la richesse des collections patrimoniales pour toucher et ramener ce vaste public auprès des acteurs traditionnels de l'étude du passé, archives, bibliothèques, universités et musées en tête.

\section{Quel usage des sources pour quelle communication?}

Que la vocation du compte Twitter ou de la page Facebook en question soit de mettre en valeur une collection patrimoniale, de servir d'exercice de médiation culturelle pour une classe d'université, de lutter pour qu'une thématique historique sorte de l'ombre, de chercher à créer une large audience pour faire la preuve de l'efficacité d'une entreprise de communication numérique, de créer des revenus publicitaires ou de rendre hommage à un grand-père tombé au combat, tous ont en commun l'utilisation de documents d'archives souvent photographiques - dans leurs messages. Or, le rôle et la place qu'occupent ces sources dans ces tweets ou posts varie beaucoup en fonction de la stratégie adoptée. C'est pourquoi nous proposons une typologie des usages de documents historiques dans la communication sur les réseaux sociaux numériques (fig. 1). Elle s'articule autour de deux grandes catégories : d'une part les communications basées sur la disponibilité d'archives et qui consistent à partager des ressources pour ellesmêmes avec un fil conducteur directement conditionné par la collection ou le dépôt (un processus archive $\rightarrow$ communication), et d'autre part les communications qui, pour illustrer un récit historique ou une thématique font appel à des documents d'archive généralement disponibles en ligne (communication $\rightarrow$ archive). Ce n'est donc pas la nature des archives sollicitées qui fait, à notre sens, la différence entre ces catégories mais plutôt leur usage, comme base ou comme outil : une même photographie d'un événement politique peut autant être partagée par une institution patrimoniale valorisant ses collections que par un collectif d'historiens spécialisés dans cet événement particulier. Cette distinction entre communication basée sur des archives et communication utilisant des archives ne renferme par ailleurs aucun jugement de valeur sur la démarche ${ }^{4}$. En effet, on trouve le meilleur comme le pire dans chacune d'entre elles : dans la première catégorie, face aux institutions qui partagent quelques pièces de leurs fonds et leurs utilisateurs qui tweetent des «perles» découvertes au fil de leurs consultations, on trouve par exemple également les usages parasites et semi-automatisés de comptes qui partagent en masse des clichés ultra-célèbres ou divertissants issus de grandes banques d'images (voire de ces mêmes institutions) dans une course effrénée à l'attention. À l'inverse,

\footnotetext{
${ }^{4}$ Pas plus qu'un jugement de valeur sur sa mise en œuvre : le référencement correct des images n'est pas un critère qui permet de différencier systématiquement les comptes officiels des comptes amateurs. Ainsi, des institutions partagent des images sans en donner la source comme des particuliers renvoient à leurs sources avec précision.
} 
dans la catégorie comprenant les usages articulés autour d'un récit particulier, on trouve autant d'excellents fils pédagogiques illustrés avec rigueur que de tentatives d'instrumentalisation de la mémoire.

Notons enfin qu'il existe un genre de communication qui se développe aussi bien dans une perspective fondée sur un corpus que sur un récit ou une thématique: l'approche événementielle (au centre de la fig. 1) qui consiste à documenter un événement, soit en suivant une source unique (auquel cas elle appartient à la première catégorie), soit en se focalisant sur le récit pour utiliser des images ou des fragments de textes sélectionnés pour l'occasion. Nous développons ces usages ci-dessous.

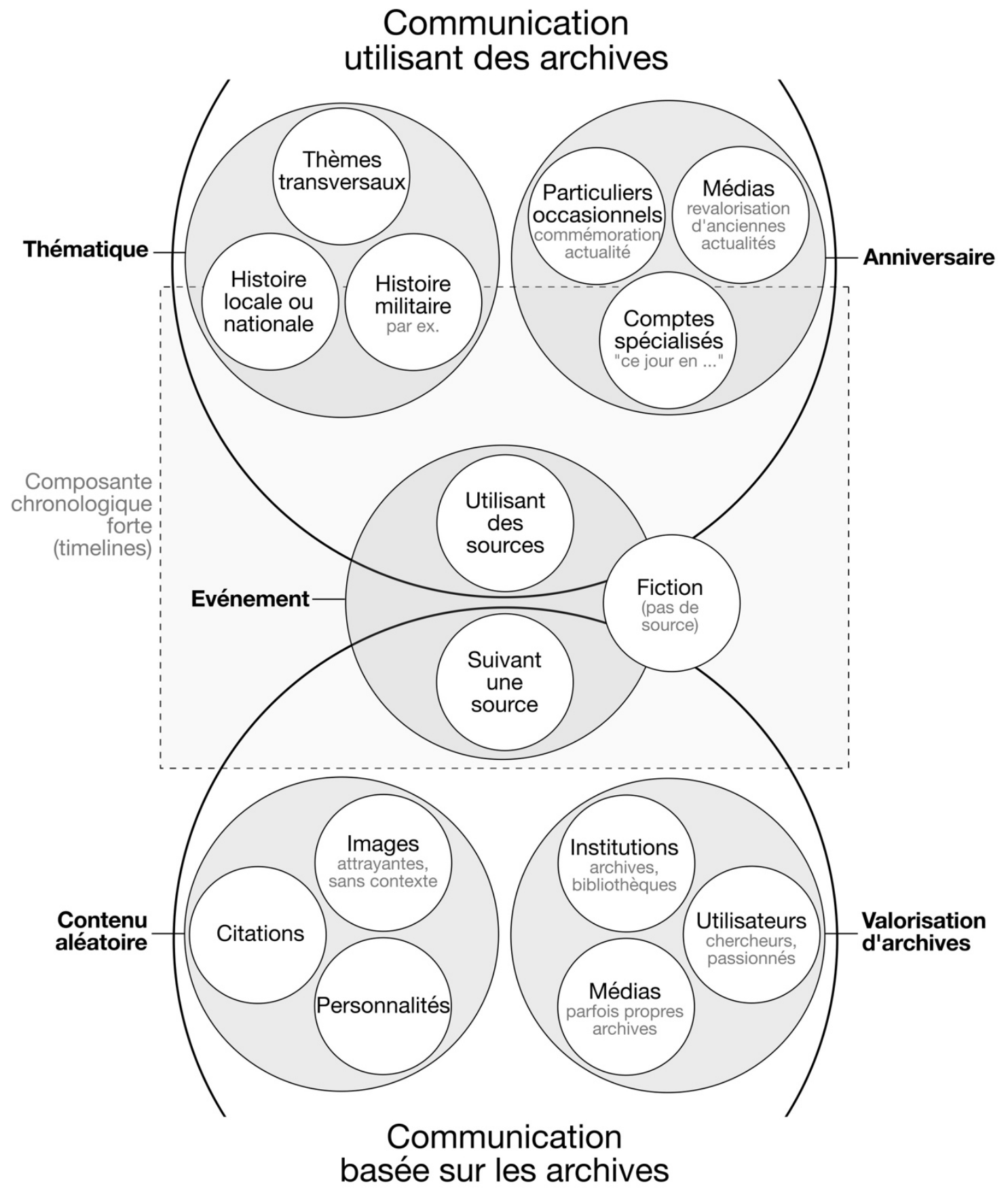

Fig 1. Typologie des usages de sources historiques sur les réseaux sociaux numériques. 


\section{Une communication basée sur des}

\section{archives}

$\mathrm{Au}$ sein de la première catégorie, qui regroupe les usages qui consistent à publier sur les réseaux sociaux numériques des photographies d'archives pour elles-mêmes, nous distinguons deux principaux types de stratégies.

Le premier (fig. 1 en bas à droite) concerne les usages professionnels ou quasi-professionnels de la part des institutions patrimoniales, de leurs utilisateurs ou des médias. Ces usages ont ceci de particulier qu'ils se basent sur des documents qui peuvent être qualifiés et contextualisés avec précision par les personnes concernées. Sans surprise parce qu'ils sont les principaux pourvoyeurs de matériel, les acteurs les plus actifs de ce genre à part entière qui consiste à valoriser des archives sont les institutions patrimoniales elles-mêmes. Or, leur travail ne se limite évidemment pas à partager des images donnant envie à leur public de visiter ses fonds, physiquement ou en ligne. Dans de nombreux cas, il s'agit de susciter une interaction qui crée le lien avec leurs utilisateurs ou de potentiels visiteurs. Ce qui est valable pour les bibliothèques traditionnelles ou les musées, qui trouvent par là un moyen supplémentaire d'entretenir une relation entre institution et usagers au travers de plateformes sociales qui complètent ainsi leur dispositif physique ${ }^{5}$, l'est également pour les

\footnotetext{
${ }^{5}$ M.-F. Audouard, M. Rimaud et L. Wiart, Des tweets et des likes en bibliothèque, Paris, Éditions de la Bibliothèque publique d'information, 2017,

https://books.openedition.org/bibpompidou/2073. Voir également, au sujet des musées N. Holdgaard et L. Klastrup «Between control and creativity: challenging co-creation and
}

archives et les bibliothèques qui rendent disponible une partie de leurs documents anciens en ligne: l'exemple de Gallica ${ }^{6}$, la bibliothèque numérique de la $\mathrm{BnF}$, est d'ailleurs un cas d'école d'animation de communauté. Dans des messages jouant souvent sur l'humour ou les qualités esthétiques des documents proposés, les éditeurs du compte Twitter de l'institution n'hésitent pas à diversifier les approches en proposant par exemple de petits concours (fig. 2 I) à leurs plus de 50000 abonnés ${ }^{7}$. Cette proactivité a pour conséquence la structuration progressive d'un réseau de «Gallicanautes ${ }^{8} »$, de super-utilisateurs qui participent activement à la diffusion et à la valorisation des archives de la BnF. C'est d'ailleurs parmi les usagers des institutions patrimoniales que l'on trouve un vivier d'internautes prompts à partager avec leurs abonnés les trouvailles dénichées en salle de consultation. Certains s'y forgent une solide réputation, à l'image de l'historien du livre de l’Université de Leiden Erik Kwakkel (@erik_kwakkel)dont les plus de 20000 abonnés peuvent suivre les pérégrinations

social media use in a museum context », Digital Creativity, 25, 3, 2014, p. 190-202.

${ }^{6}$ https://gallica.bnf.fr/

${ }^{7}$ Soit quatre fois moins que le compte officiel de la $\mathrm{BnF}$ (@laBnF), mais un « engagement » bien supérieur puisque les messages de Gallica génèrent un nombre d'interactions qui se situe dans la plupart des cas dans une fourchette 20 et 200 retweets et likes contre 5 à 20 pour le compte principal de l'institution. À l'heure des comparaisons entre nombre de followers, cette mesure de l'engagement est importante : le compte officiel de la Bibliothèque du Congrès (@librarycongress) compte 1.2 million d'abonnés pour un « engagement moyen » inférieur à Gallica. Pour permettre la comparaison, nous appliquons une telle mesure d'« engagement » moyen à la plupart des comptes Twitter évoqués ci-après, sur la base des statistiques observées au printemps 2018 (la fourchette de valeurs indiquant l'intervalle indicatif dans lequel on trouve la majorité des valeurs, certains tweets sur- ou sous-performants pouvant parfois se situer en dehors).

${ }^{8}$ https://gallica.bnf.fr/html/und/du-cote-des-gallicanautes 
médiévales9. À lui seul, il rassemble une communauté d'amateurs et de passionnés de paléographie et de codicologie, avec un accent particulier sur les éléments les plus visuels, enluminures originales en tête (fig. $2 \mathrm{~J}$ ). Un autre groupe d'acteurs participe également à cette démarche de valorisation, celui des médias qui relaient souvent volontiers des pépites mises en évidence par les institutions patrimoniales (exemple fig. 2 E) ou piochent tout simplement dans leurs propres archives.

Mais dans ce même ensemble de propagateurs de documents historiques, on trouve également une myriade de comptes qui fondent leur audience sur le partage de photographies de célébrités, de citations inspirantes ou d'images bien connues ou volontairement surprenantes (fig. 1 en bas à gauche). Même si la démarche est naturellement bien différente de celle des institutions d'archives et de leurs utilisateurs, elle repose fondamentalement sur le même principe: ces sources existent et sont à disposition, les partager pour elles-mêmes (sans suivre un fil rouge thématique, par exemple) crée de l'« engagement». Or, comme on le verra plus loin, cet engagement n'a pas du tout les mêmes fins que celui des acteurs traditionnels qui visent surtout à étendre la diffusion de leurs propres contenus et à les faire découvrir au plus grand nombre tout en fédérant une communauté d'utilisateurs. Tout en remarquant donc que cette démarche bien que

\footnotetext{
${ }^{9}$ E. Kwakkel, «A Window Display of 140 Characters: Why and How Twitter Works for Me as an Academic », medievalfragments, 11/08/2012,

https://medievalfragments.wordpress.com/2012/08/11/awindow-display-of-140-characters-why-and-how-twitterworks-for-me-as-an-academic/. Son taux d'engagement est relativement similaire à celui de Gallica.
}

similaire sur la forme ne fait pas de ces comptes un échelon logique du développement de la communication des institutions patrimoniales, leur succès interroge. Que cherchent les centaines de milliers d'abonnés - voire les millions pour certains - de ces comptes qui publient à un rythme soutenu des portraits des Beatles (fig. 2 A) ${ }^{10}$, des citations célèbres (fig. 2 B) ou des clichés du débarquement de Normandie (fig. 2 C) ${ }^{11}$ ? Sans pour autant postuler que tous ces comptes soient mis en place comme on le verra plus loin dans le seul but de générer un quelconque profit puisqu'on ne peut pas exclure que certains d'entre eux soient alimentés par des passionnés d'histoire spectaculaire, leur usage des documents historiques comme moyen de divertissement est dangereux, à terme, pour le développement d'une «histoire publique » riche et diversifiée. La fig. $2 \mathrm{~F}$ résume à elle seule tout un pan de l'Internet en faisant la synthèse entre la fascination pour les chats ${ }^{12}$ et la réappropriation de contenus, historiques ou pas, sans considération pour le contexte et le crédit de l'auteur. Et même si certains, alarmés par ce constat cherchent à corriger ces défauts, par exemple en créditant les images partagées par ces comptes peu scrupuleux (fig. $2 \mathrm{G})^{13}$, ces initiatives ne résistent généralement pas au rythme de

\footnotetext{
${ }^{10}$ Le compte@History_Pics a très récemment été suspendu par Twitter. Il comptait un peu plus de 4 millions d'abonnés et générait en moyenne entre 1000 à 5000 engagements par tweet.

${ }^{11}$ Le compte@HistoryInPics publie deux tweets par jour à destination de ses 4.14 millions d'abonnés, générant lui aussi un engagement moyen de 1000 à 5000 interactions (retweets et likes) par message.

${ }^{12}$ E. Podhovnik, «The Meow Factor - An Investigation of Cat Content in Today's Media », Proceedings of the Arts \& Humanities Conference, Venice, 2016, p. 127-139.

${ }^{13}$ Le compte@CreditsInPics, qui renseigne l'auteur et redirige vers la version originale d'une image partagée par @HistoryInPics est désormais inactif.
} 
publication très soutenu et à la multiplication des comptes de divertissement. Au-delà de très fréquentes erreurs ou imprécisions, ces comptes

$$
\text { 당. ClassicPics }
$$

They did it before it was cool
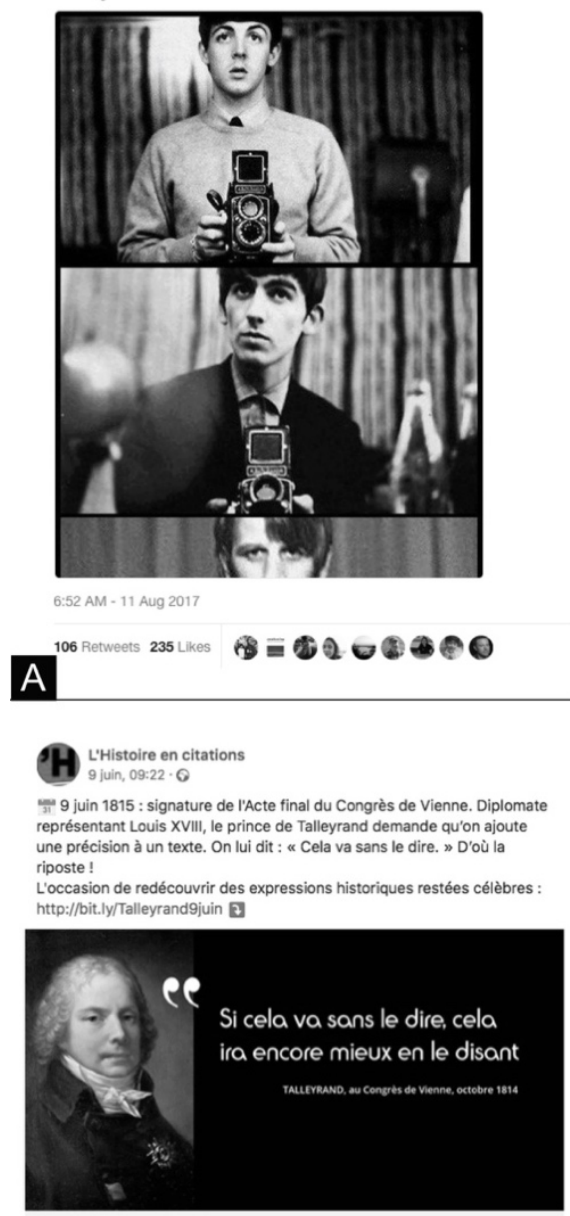

HISTOIRE-EN-CITATIONS FR

9 juin 1815 : signature de l'Acte final du Congrès de Vienne Cité en français, ce mot figure dans beaucoup de dictionnaires étrangers.

\section{B}

\section{History In Pictures}

A landing craft approaching Omaha Beach in Normandy, France on the 6th June 1944 also known as D-Day.

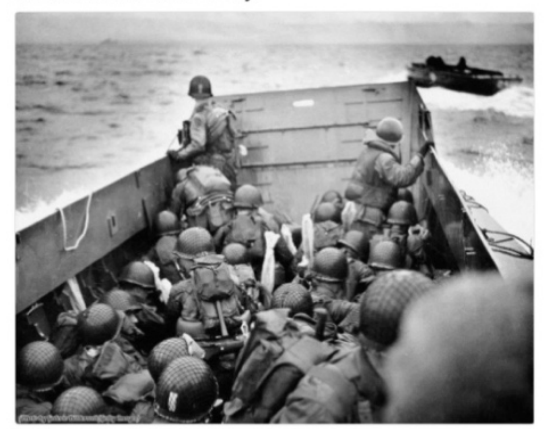

2:29 AM - 26 Jan 2017

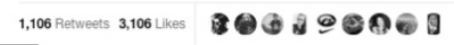

C

\section{A-. BETHUNE 1418}

Cette nuit, 6 bombes sont tombés sur Béthune

ST Transiate from Firench

D

\section{(J) Le Temps *}

Quand I'Europe naissait à Genève, en 1929 letemps.ch/monde/2017/08/...

o Transiate Twoet

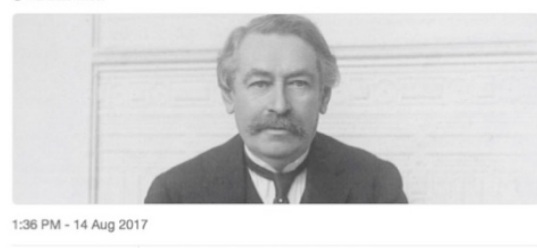

\section{$\mathrm{E}$}

\section{$\stackrel{10.1}{\rightleftharpoons} \frac{\text { ClassicPics }}{\text { eHistory_Pics }}$}

A dog and cat in Paris

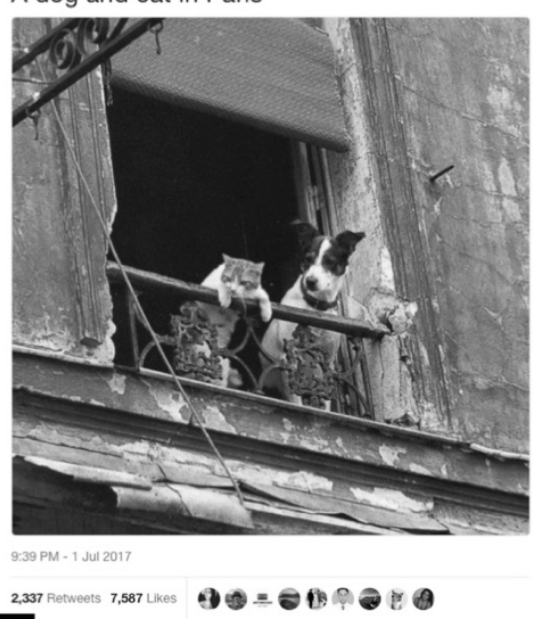

F

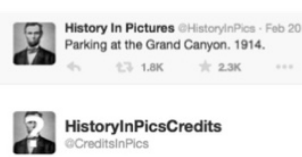

Narrated by O.K. Parker here: photographicexploration.com/mertz.html "@HistorylnPics Parking at the Grand Canyon. 1914. "

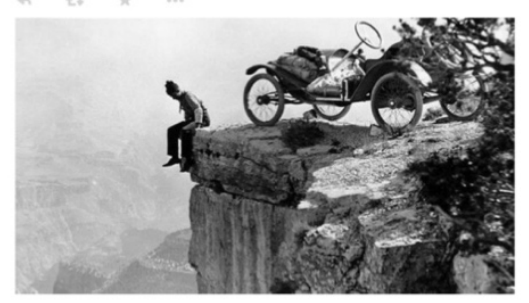

G

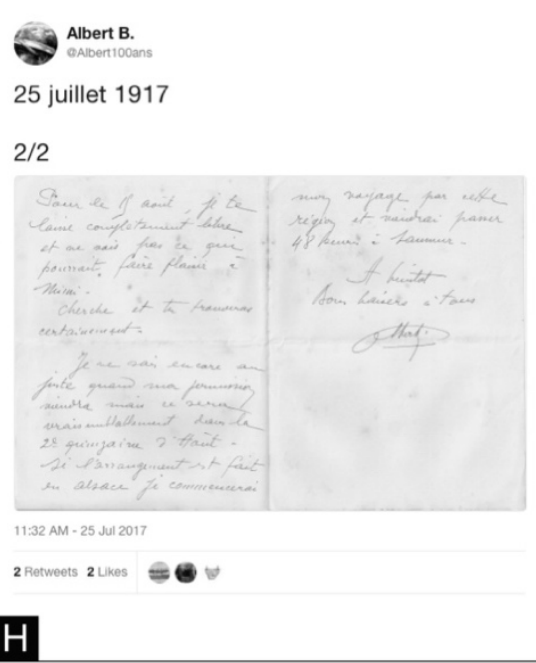

GallicaBnF o
GGallicaBnF

On a retrouvé Elvis Presley ! c.bnf.fr/fQU \#ElvisIsAlive \#TrouveUnSosieDansGallica

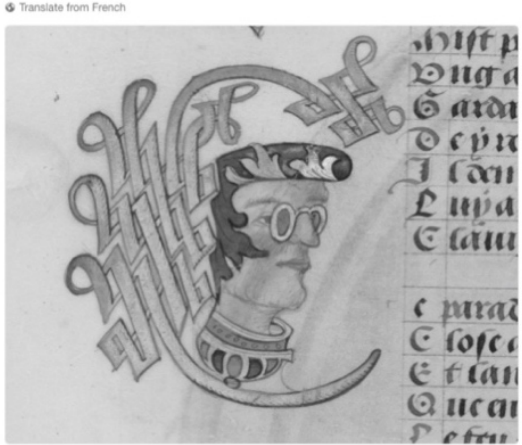

4:16 PM- 28 Jul 2017

183 Retweets 283 Lhes है।

I

\section{1}

9. Erik Kwakkel

This scribe, who identifies himself as Nicolaus, made sure to point out - literally that he copied it (. Vendôme, Bibl. mun., ms. 0245).

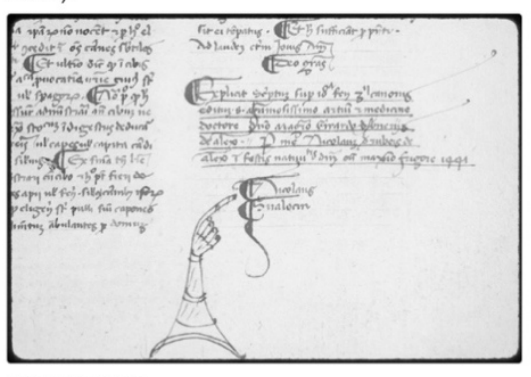

$\sqrt{1}$

Fig 2. Exemples de comptes Twitter et Facebook illustrant différentes démarches « archives $\rightarrow$ communication ». 


\section{Un récit qui utilise des archives}

Notre seconde catégorie d'usages, qui regroupe les démarches consistant à élaborer un discours pour l'illustrer ensuite au moyen de documents d'archives (communication $\rightarrow$ archive), se divise également en plusieurs sous-ensembles.

On y trouve tout d'abord les fils consacrés à des thématiques particulières (fig. 1 en haut à gauche). C'est par exemple le cas lorsqu'une association spécialisée ou une société savante communique sur l'actualité de sa discipline, l'histoire culinaire par exemple (fig. $3 \mathrm{~K})^{14}$, ou une focale locale ou géographique (fig. $3 \mathrm{M}$ ), sans nécessairement donner à ses messages un caractère chronologique. C'est également le principe adopté par des blogueurs, passionnés ou enseignants-chercheurs lorsqu'ils déroulent leurs successions de tweets consacrés à leurs recherches, illustrées par des photographies, des extraits de sources ou du matériel audio-visuel (fig. $3 \mathrm{~L})^{15}$. À la différence des usagers partageant leurs trouvailles en archive, et bien qu'il puisse s'agir des mêmes personnes, le processus est ici bien différent puisqu'il ne s'agit plus de publier des documents pour eux-mêmes mais d'élaborer un storytelling puis de partir à la recherche de ressources à mobiliser dans ce cadre.

Format particulièrement efficace et très impliquant pour son public qui se voit projeté dans le passé en raison de la proximité de calendrier, les contenus

\footnotetext{
${ }^{14}$ Le compte Twitter des «Culinary Historians » de Californie du Sud (@CHSoCal) compte près de 3500 abonnés et génère entre 0 et 10 engagements par tweet en général.

${ }^{15}$ En plus de la qualité de ses threads très bien documentés, l'approche politisée de l'historienne des mouvements révolutionnaires Mathilde Larrère, suivie par près de 40000 abonnés sur Twitter (@LarrereMathilde), explique la viralité de certains de ses messages.
}

liés à un anniversaire (les fameux «this day in...» ou « on this day», par exemple, voir fig. 1 en haut à droite) sont très populaires sur des médias dont l'immédiateté est la marque de fabrique. Si cette démarche attire beaucoup de comptes à faible valeur ajoutée proches des propagateurs très généralistes évoqués plus haut (fig. $3 \mathrm{R})^{16}$, elle permet également à des institutions patrimoniales de valoriser leurs collections de manière originales, à l'image du compte@TodaysDocument qui diffuse à ses 46000 abonnés ${ }^{17}$ des documents des archives nationales américaines (fig. $3 \mathrm{O}$ ) ou d'autres initiatives basées sur les archives de médias (fig. $\left.3 \mathrm{P}^{18}\right)^{19}$.

Mais alors qu'il apparaît que les contenus qui immergent leur public dans une expérience quasi «vécue» de l'histoire connaissent un succès important, c'est surtout lorsqu'ils sont intégrés dans une démarche chronologique à plus long terme qu'ils révèlent l'intérêt des réseaux sociaux numériques comme lieu d'un véritable récit. Le cas le plus emblématique et qui figure parmi les premiers du genre est le compte Twitter @ RealTimeWWII (fig. 3 N). Lancé en 2011 et comptant sept ans plus tard plus d'un demi-million

\footnotetext{
${ }^{16}$ Suivi par 45000 abonnés, le compte Twitter @WorldHistory101 automatise désormais ses messages en renvoyant chaque jour vers des sites internet compilant des événements s'étant déroulés le jour même. Son impact auparavant important a fortement décru ces dernières années depuis l'abandon de publications plus imagées (la répétitivité de ces contenus a probablement eu raison de la curation manuelle).

${ }^{17}$ De 10 à 150 engagements par tweet en général.

${ }^{18}$ Bien qu'il en utilise les archives, il ne s'agit pas d'une initiative du New York Times mais d'un projet chapeauté par Russia Today pour les commémorations de la révolution de 1917.

${ }^{19}$ Même si elle n'implique pas un recours aux réseaux sociaux numériques, notons également l'existence de la page «Unes du jour » de Gallica, générée quotidiennement : https://gallica.bnf.fr/UnesDuJour.
} 
d'abonnés, il live-tweet la Seconde Guerre mondiale à coup de plusieurs micro-messages par jour comme si elle se déroulait en direct ${ }^{20}$. Il s'agit surtout d'un des exemples les plus populaires ${ }^{21}$ et les plus abouti de storytelling historique sur les réseaux sociaux numériques puisqu'il implique une curation et une synthèse importante tout en faisant un usage très riche des ressources iconographiques disponibles en ligne ${ }^{22}$. Démarche générique pouvant être appliquée à de nombreux autres objets historiques se déroulant pendant une période suffisamment longue pour que le processus ait de l'intérêt, le principe de faire «revivre» un événement développé par @ RealTimeWWII ${ }^{23}$ a été depuis récupéré dans une grande variété de contextes, de l'histoire militante de la gauche radicale (fig. 3 S) à l'exercice universitaire reporté sur la Première Guerre mondiale (fig. 3 Q), avec dans ce dernier cas une

\footnotetext{
${ }^{20}$ À propos de ce compte Twitter et d'autres initiatives en direct, voir E. Butticaz, «Twitter, cette machine à remonter le temps », Le Temps, 2013, https://www.letemps.ch/nosection/2013/11/22/twitter-cette-machine-remonter-temps, M. Steffen et P. Nunes Coelho, «Tweeting during World War II », H-Europe, 2014, http:/ / h-europe.uni.lu/?p=2037, ou encore V. Varin, «Tweeps Discover the Past»,

Perspectives on History, 2014,

https://www.historians.org/publications-and-

directories/perspectives-on-history/april-2014/tweeps-

discover-the-past. À noter que le projet dispose de sa propre notice sur l'encyclopédie participative Wikipédia (https:/ / en.wikipedia.org/wiki/RealTimeWWII) et que l'on trouve une documentation abondante à son sujet dans les médias.

${ }^{21}$ De 200 à 1000 engagements par tweet en général.

${ }^{22}$ L'exemple de la fig. $3 \mathrm{~N}$ montre les limites d'un tel usage illustratif : la photographie représente un soldat suisse surveillant le col du Simplon, à la frontière avec l'Italie et pas la France.

${ }^{23}$ Qui en est d'ailleurs à sa $2^{\mathrm{e}}$ diffusion puisque créé à l'été 2011 en synchronisation avec 1939 (1945 a donc été atteint en 2017).
}

attention particulière à la documentation de chaque information $^{24}$.

Bien sûr, et c'est la limite d'une telle typologie, l'approche événementielle (fig. 1 au centre) n'est pas uniquement le produit d'une démarche «communication $\rightarrow$ archive » puisqu'il se trouve dans certains cas des sources suffisamment complètes et suivies pour les utiliser elles-mêmes comme base du récit. La récente vague de commémorations de la Grande Guerre a par exemple provoqué la publication sur Twitter de nombreux journaux intimes de soldats français: rédigés au jour le jour, ils constituent un matériel parfait pour une mini-chronique s'étendant sur une temporalité de plusieurs années, parfois enrichie de photographies des pages elles-mêmes ou d'autres documents d'illustration. L'exemple d'« Albert B. » (@Albert100ans) et ses près de 3000 abonnés, bien que bénéficiant d'une réception relativement modeste $^{25}$, est particulièrement méthodique dans sa référence aux sources et est donc autant une réflexion archivistique sur les supports physiques qu'une fenêtre ouverte sur le quotidien pendant le conflit (fig. $2 \mathrm{H}$ ). Dans le même contexte mémoriel, d'autres sources - plus sérielles encore, et donc automatisables - sont mises à contribution, comme les documents du Cabinet de guerre de Londres (@UKWarCabinet), les journaux des unités britanniques (@UnitWarDiaries) ou encore les comptes rendus de la petite ville de Béthune, particulièrement éprouvée par la guerre (@Bethune1418,voir fig. 2 D). Ces initiatives, moins imagées que les récits

\footnotetext{
${ }^{24}$ "World War One goes Twitter», Master in European Contemporary History, Université du Luxembourg, http://heurope.uni.lu/?page_id=62.

${ }^{25} \mathrm{Il}$ génère entre 3 et 10 engagements par tweet en général.
} 
illustrés par des documents iconographiques, trouvent moins facilement leur public mais sont néanmoins des tentatives intéressantes de renouvellement de la communication historique ${ }^{26}$. Le suivi en direct d'événements ancrés dans l'histoire ne manque pas non plus d'inspirer des initiatives se détachant cette fois-ci totalement des sources pour donner lieu à des récits fictionnels ou humoristiques. Au registre des projets découlant d'une stratégie de communication reconstituant un événement historique, la commémoration du centenaire de la croisière inaugurale du RMS Titanic en 2012 au moyen du compte Twitter @TitanicRealTime par l'éditeur The History Press est particulièrement immersif (il a réitéré l'expérience en 2013 puis 2014 et a depuis été imité par plusieurs autres comptes) ${ }^{27}$. Sa particularité est de se présenter sous la forme d'un récit à plusieurs voix, du capitaine à l'équipage ou aux passagers en passant par le navire Carpathia qui reçoit son message de détresse (fig 3 T). De son côté, le projet du Musée de la Grande Guerre de Meaux consistant relater la vie des tranchées de 1914 au travers d'un personnage créé pour l'occasion sur Facebook, «Léon Vivien ${ }^{28}$ », brouille encore plus la distinction entre histoire et fiction ${ }^{29}$. Original par sa forme, puisqu'il simule l'existence d'un réseau social numérique au début du $\mathrm{XX}^{\mathrm{e}}$ siècle avec des

\footnotetext{
${ }^{26}$ Suivi par près de 12000 abonnés, @ukwarcabinet ne génère quasiment pas d'engagement, tout comme @unitwardiaries et ses 1000 abonnés. Malgré plus de 3000 tweets, le compte@bethune1418 est très peu suivi (200 abonnés) et ne crée pas non plus de dynamique autour de ses publications très sérielles.

${ }^{27}$ Le compte rassemble plus de 50000 abonnés et génère en moyenne entre 20 et 100 engagements par message.

${ }^{28}$ https://www.facebook.com/leon1914/

${ }^{29}$ B. Texier, « Léon Vivien, un poilu sur Facebook ! , Archimag, 23.05.2014, http://www.archimag.com/archivespatrimoine/2014/05/23/leon-vivien-poilu-facebook.
}

personnages qui se répondent sous les yeux des 60000 internautes abonnés à la page Facebook en question, cette sorte de journal intime pose un problème de rapport aux sources puisqu'en plus d'inventer logiquement le récit, il est illustré par des documents d'archives dont certains sont retouchés pour mettre en scène le personnage fictif dont le visage a été recomposé sur la base des portraits de l'équipe de communication en charge de l'opération. Toujours est-il que le succès du projet en fait un excellent exemple de communication historique: une exposition médiatique maximale pour le musée, une baisse de la moyenne d'âge de son public et la publication des images produites à cette occasion dans un $\operatorname{livre}^{30}$, comme pour conclure que le numérique doit revenir à un support plus traditionnel pour être valorisé dans la durée.

\footnotetext{
${ }^{30}$ Collectif, Léon Vivien 1914, le poilu aux 60000 fans, Paris, Editions de l'Opportun, 2013.
} 
Cullinary Historians echsocal Sep 13
The concept of the velvet rope began at The Trocadero in the 1930s

C. Cullinary Historians ECHSoCal $\cdot$ Sep 13
In 1920 s Hollywood $66 \%$ of films stowr

2 Culinary Historians $\mathrm{CCHSOCal} \cdot \mathrm{Sop}$ ? 31 K partying antics... but with no one watching no one came so it closed

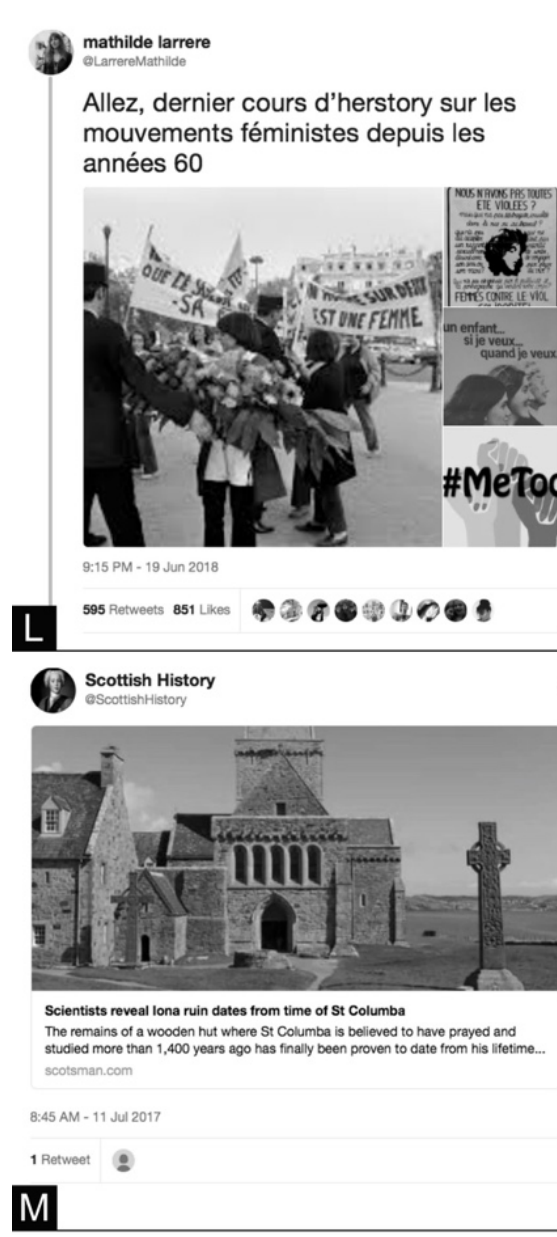

I. WW2 Tweets from 1942 eRoumimowWI - Sop 30
$\sim 20,000$ Jewish \& anti-Nazi refugees now shelter in Switzerland, some
still smuggled in by sympathetic Swiss- but 1000 s more forced away. still smuggled in by sym

W. WW2 Tweets trom 1942 efearimenwil - Sop 30

Swiss government decides not to admit Jews fleeing Nazi-occupied France; border guards ordered to turn back refugees.

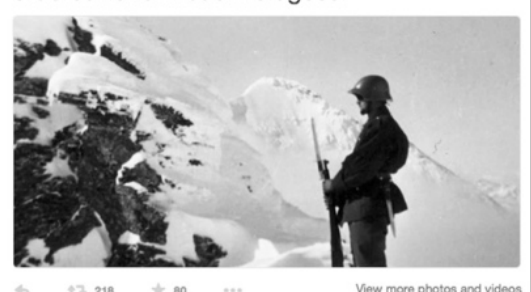

N

Fig 3. Exemples de comptes Twitter et Facebook illustrant différentes démarches « communication $\rightarrow$ archives ».

\section{(3.) World History 101}

On this day in 1929, the Graf Zeppelin left Lakehurst, New Jersey, on the first roundthe-world trip by an airship. week of $6 / 21 / 1943$ details Allied advances in the Mediterranean prior to the invasion of Sicily, 75 years ago. "Disease in the Tropics" featured on the reverse.

catalog.archives.gov/id/66395147 \#WWII

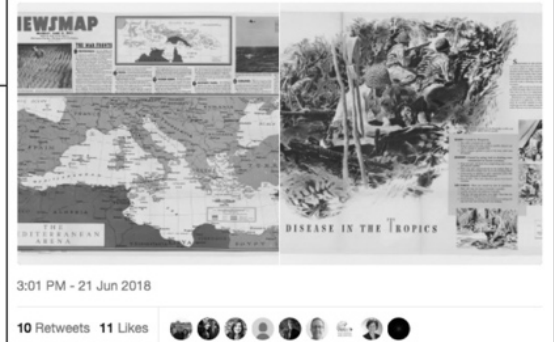

O

the the New York Times.

Vital Need of Victory as Gompers Sees it; Labor Leader Assails the Internationalists

Who Would Prevent Pushing the War to a Definite Decision Against Kaiserism \#1918LIVE

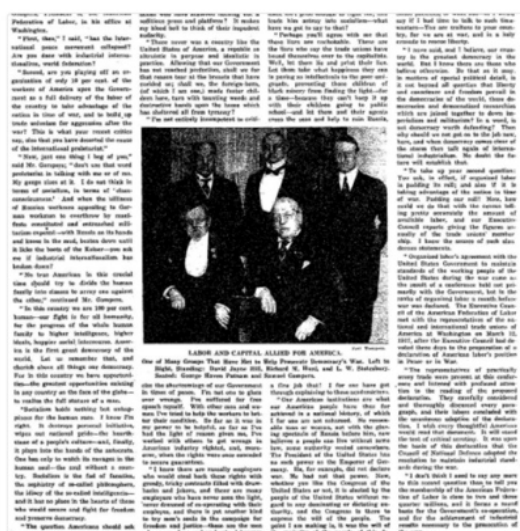

P

Tweets from WW1

Brit. royalty on the West.Front (1/2): Queen Mary of Teck talks to a French worker repairing Brit. uniforms, \#Rouen. goo.gl/8LpUfz

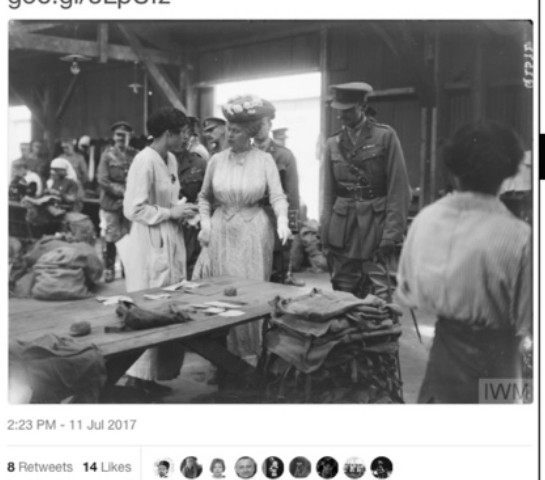
everywhere we find them. It's agony for me to sit on a horse, but I'm managing.

1:26 AM - 21 Jun 2018

U

7. DalilyRadical History Eradicaldalily - OCt 14

Penspiring to a

2. DailyRadical History eraficaldaily - Oot 14

Oct 141981 - Dock workers in Darwin, Australia start 7-day strike, refusing to load uranium on board "Pacific Sky" for US military use.

DailyRadical History Pradiadienty $\mathrm{Oen} 14$

Oct 141817 - Marcus Moller Thrane born. Norwegian author, journalist \& leader of 1st Norwegian labour movement known as the Thrane

S

\section{[] TitanicVoyage}

*Carpathia Calling Titanic with zero

response, we can only hope their wireless is damaged and nothing worse has happened.

4:42 AM - 15 Apr 201.

\section{$\mathrm{T}$}

\section{Jehan le Brave}

Reçois nombre covrriels Apothicaires sovcievx de me confier movlt Ecvs via Vnion Occident. N'y entends govtte.

STinestan Thes

4:33 PM - 26 Mar 2018

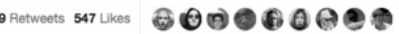

V

\section{Michael V Calaphates}


Témoignant à leur manière de l'intérêt des internautes pour les contenus historiques, les détournements humoristiques de cette démarche de récit jour après jour reçoivent parfois un accueil plus enthousiaste que les fils à vocation plus archivistique ou commémorative.

C'est par exemple le cas du compte Twitter @ CryForByzantium qui retrace de manière très vivante un millénaire d'histoire byzantine (fig. 3 U). Malgré l'absence de sources ou de références dans les micro-messages, son ton très décalé et sa régularité (entre deux et quatre tweets par jour) en font un outil de vulgarisation qui fait figure de porte d'entrée originale vers cette thématique pour presque dix mille internautes ${ }^{31}$.

Sans pouvoir lister tous les exemples de récupération humoristique, des comptes comme @ RealHistoryPic, qui se livre à une satire de l'histoire et de la politique indienne en inventant de nouvelles légendes ${ }^{32}$, ou «Jehan le Brave» (@Jehanlb), qui égaie le fil d'actualité de ses près de 40000 abonnés avec des tweets tout droit sortis du Moyen-Âge (fig. $3 \mathrm{~V}$ ) 33 , illustrent bien la diversité et la créativité de cette dynamique.

\section{L'envers du décor}

Finalement, et pour reprendre l'expression de « mise en scène » de l'histoire, que savons-nous sur les personnes qui œuvrent dans les coulisses des différents projets abordés ci-dessus? Encore une

\footnotetext{
${ }^{31}$ Voir également le blog du projet : https://palaiologinos.wordpress.com. Les messages de ce compte Twitter génèrent un engagement qui se situe généralement entre 20 et 40 retweets et likes.

${ }^{32}$ Le compte est suivi par 150000 abonnés, son engagement moyen se situe entre 200 et 3000 interactions.

${ }^{33}$ Entre 200 à 1000 engagements par tweet en général.
}

fois, la réponse est à l'image de la diversité des approches : alors que les comptes institutionnels ou professionnels sont relativement transparents sur leur fonctionnement, l'envers du décor des comptes pratiquant une forme de vulgarisation aveugle et automatisée est beaucoup plus difficile à cerner, ceci d'autant plus lorsqu'il s'agit d'une démarche commerciale.

Parmi les usages les plus classiques, sans revenir sur les exemples de communications en provenance d'institutions patrimoniales, de médias ou d'associations scientifiques dont les rouages sont généralement bien connus ou tout simplement déductibles des stratégies retenues pour leur présence sur les réseaux sociaux numériques, le cas des acteurs individuels, qu'ils soient chercheurs ou passionnés, retient notre attention. En la matière, les expériences d'Erik Kwakkel et de Mathilde Larrere ont pour point commun le fait que leur propre découverte de l'intérêt de leurs abonnés pour leurs publications historiques a eu lieu dans un second temps. Dans le premier cas, la vocation du chercheur ne s'est révélée qu'après avoir constaté l'attention de sa communauté pour des photographies de manuscrits partagées occasionnellement ${ }^{34}$. Dans le second, "l'historienne a pris le pas sur la militante ${ }^{35}$ » après s'être rendu compte de la nécessité de ne pas se cantonner à la revendication mais de développer de véritables fils bien documentés. Dans ces situations, c'est donc assez naturellement et sans calcul stratégique que ces

\footnotetext{
${ }^{34}$ E. Kwakkel, «A Window Display of 140 Characters », op. cit., voir également les autres billets de son carnet de recherche.

${ }^{35}$ V. Servat, « Faire de l'histoire sur Twitter ? Entretien avec @LarrereMathilde », Devenir historien-ne, 2016, https://devhist.hypotheses.org/3336.
} 
réseaux sociaux numériques ont évolué d'un lieu d'expression personnelle à une caisse de résonnance pour toute une communauté.

Le compte@RealTimeWWII doit également sa création à un passionné d'histoire, Alwyn Collinson. Moins d'un mois après le lancement de son fil d'actualités suivant le déroulement des événements de la Seconde Guerre mondiale, l'ancien étudiant d'Oxford est repéré par le site spécialisé en nouvelles technologies The Next Web qui lui consacre un article ${ }^{36}$, faisant passer sa communauté d'abonnés de quelques centaines à plus de 10000 en quelques jours. La progression est fulgurante puisque deux mois plus tard, alors que le New York Times s'intéresse à son tour à la popularité de ce projet qui donne déjà lieu à des traductions en plusieurs langues et à d'autres livetweets historiques sur des thématiques connexes, celui-ci compte désormais plus de dix fois plus d'abonnés ${ }^{37}$.

Mais tous les animateurs de communautés ne sont manifestement pas animés de la même passion pour l'histoire. Une étude plus approfondie du comportement des principaux comptes Twitter et Facebook partageant en masse des photographies d'archives décontextualisées nous montre qu'audelà de quelques enthousiastes, les personnes qui passent une partie de leur temps à rédiger des légendes accrocheuses à destination de millions d'internautes peuvent être séparés en quatre

\footnotetext{
${ }^{36}$ P. Sawers, « How an ex-History student is using Twitter to bring World War 2 to life », The Next Web, 25.09.2011, https://thenextweb.com/twitter/2011/09/25/how-an-exhistory-student-is-using-twitter-to-bring-world-war-2-tolife/.

${ }^{37}$ J. Schuessler, «The Tweets of War: What's Past is Postable », The New York Times, 27.11.2011, https://www.nytimes.com/2011/11/28/arts/re-enactinghistorical-events-on-twitter-with-realtimewwii.html.
}

catégories qui parfois s'entremêlent et se complètent. On trouve tout d'abord des individus pris de passion pour les réseaux sociaux numériques sans s'intéresser à la matière ellemême et qui prennent le média comme un terrain de jeu. L'influence est dès lors un but en soi, sans nécessairement qu'elle génère un bénéfice financier. C'est le cas, dans un premier temps, des fondateurs de@HistoryInPics qui a longtemps été le compte Twitter le plus suivi dans la galaxie des comptes historiques : deux adolescents créant des chaînes YouTube, des pages Facebook et des comptes Twitter en masse, sur des sujets variés mais toujours choisis pour susciter l'adhésion d'un public qui apprécie partager de temps en temps une belle photographie de paysage, d'animal mignon ou d'événement historique ${ }^{38}$. Ces comptes renforçant leurs bases de «fans» en se faisant mutuellement de la publicité, leurs audiences atteignent parfois plusieurs dizaines de millions d'abonnés. La tentation de la monétisation n'est pas loin, c'est la deuxième catégorie : des comptes qui profitent de tels taux d'engagement pour placer de temps en temps des publicités sur commande, « retweetent» le message d'une marque partenaire, voire prévoient avec elle une campagne de native advertising consistant à promouvoir discrètement leurs produits sous couvert de coller à la ligne éditoriale habituelle du compte en question (une publicité vintage pour des cigarettes ou une boisson alcoolisée qui veut ainsi rappeler son ancienneté à ses consommateurs, pour ne prendre que des

\footnotetext{
${ }^{38}$ C. Madrigal, «The 2 Teenagers Who Run the Wildly Popular Twitter Feed @HistoryInPics », The Atlantic, 23/01/2014, https://www.theatlantic.com/technology/archive/2014/01/ the-2-teenagers-who-run-the-wildly-popular-twitter-feedhistoryinpics/283291/.
} 
exemples observables dans les fils historiques). Les deux catégories suivantes, plus rares, font un usage beaucoup plus détourné des audiences massives créés par ces comptes historiques. L'une rassemble tout simplement des agences de communication numériques qui font vis-à-vis de leurs clients la preuve de leur capacité à créer une campagne à succès. L'autre, des agences ou des particuliers dont la finalité est de faire prendre de la valeur à des comptes Twitter ou Facebook pour les revendre ensuite à des individus ou des entreprises prêts à payer pour se lancer sur les réseaux sociaux numériques avec plusieurs milliers ou centaines de milliers d'abonnés ${ }^{39}$.

\section{Conclusion}

Constatant qu'indépendamment de sa qualité, l'efficacité de l'«engagement» des stratégies de communication historique semble être assez directement déductible de la typologie détaillée cidessus, faut-il en conclure que l'avenir de la conscience du «temps long» sur les réseaux sociaux numériques passe nécessairement par l'adoption d'un mode de communication viral et simpliste ? S'il n'est pas le lieu ici de proposer des recommandations, nous espérons que la synthèse des pratiques effectuée dans ces pages, tout en permettant de prendre la mesure de la concurrence à laquelle sont confrontées les initiatives de médiation culturelle, a le mérite d'ouvrir par ricochet le débat sur la question du public et de ses

\footnotetext{
${ }^{39}$ En changeant le nom du compte par la suite et en tablant sur le fait qu'une grande partie des followers étant inactifs cette modification ne fera que légèrement baisser leur nouvelle «base de fans».
}

attentes. En effet, alors que les médias sociaux numériques peuvent être comme on l'a vu les vecteurs d'une histoire spectacle décontextualisée et instrumentalisée, ils peuvent également favoriser une démocratisation et une réappropriation de l'histoire par un public d'une très grande diversité. Dès lors, s'il semble vain de lutter contre des mauvaises pratiques sur lesquelles la communauté des historiens et des institutions patrimoniales n'a pas prise, cerner ces phénomènes permet aussi et surtout de préparer la réplique, rigoureuse, critique et créative. 